DOI: $10.1590 / 1089-6891 v 16 i 425538$

PRODUÇÃO ANIMAL

\title{
FONTES DE PÓLEN UTILIZADAS POR Apis mellifera LATREILLE NA REGIÃO SEMIÁRIDA
}

\author{
POLLEN SOURCES USED BY Apis mellifera LATREILLE IN THE SEMIARID \\ REGION
}

\author{
Shirley Nascimento Costa ${ }^{1}$ \\ Rogério Marcos de Oliveira Alves ${ }^{2}$ \\ Carlos Alfredo Lopes de Carvalho ${ }^{3}$ \\ Pamela de Jesus Conceição ${ }^{3^{*}}$
}

\author{
1 Universidade Estadual de Santa Cruz, Ilhéus, BA, Brasil. \\ ${ }^{2}$ Instituto Federal Baiano, Salvador, BA, Brasil. \\ ${ }^{3}$ Centro de Ciências Agrárias, Ambientais e Biológicas, Universidade Federal do Recôncavo Baiano, Cruz \\ das Almas, BA. Brasil. \\ *Autora para correspondência: pamellajc02@hotmail.com
}

\section{Resumo}

O objetivo deste estudo foi conhecer as fontes de pólen utilizadas por Apis mellifera L. no município de Itaberaba, Estado da Bahia. Amostras de pólen foram obtidas por meio de coletores instalados na entrada de dez colônias de $A$. mellifera, implantadas em apiário localizado em área aberta de caatinga, nos meses de dezembro/2006, janeiro, março e dezembro/2007, período no qual foi observada elevada florada na região. Foram realizadas análises quantitativas e qualitativas no laboratório de Entomologia do Centro de Ciências Agrárias, Ambientais e Biológicas da Universidade Federal do Recôncavo da Bahia, em Cruz das Almas. A análise dos resultados mostrou a presença de 78 tipos polínicos, pertencentes a 24 famílias botânicas. A família Fabaceae-Mimosoidea foi a que apresentou um maior número de tipos polínicos. Os resultados demonstraram que a espécie $A$. mellifera apresenta hábito generalista e as famílias Fabaceae-Mimosoidea, Asteraceae, Commelinaceae e Poaceae são as principais fontes na região.

Palavras-chave: abelha melífera; fontes de pólen; tipos polínicos.

\begin{abstract}
This study aimed at identifying the pollen sources used by Apis mellifera L. in the municipality of Itaberaba, State of Bahia. Pollen samples were obtained through collectors installed at the entrance of ten A. mellifera bee colonies, implanted in an apiary at a savanna area, in the months of December of 2006, January, March and December of 2007, period in which high blooming was observed in the area. The quantitative and qualitative analyses were accomplished at the laboratory of Entomology of the Center of Agrarian, Environmental and Biological Sciences of Universidade Federal do Recôncavo da Bahia, County of Cruz das Almas, State of Bahia, Brazil. Through the analysis of the obtained results, we verified the presence of 78 pollen types, belonging to 24 families. The family Mimosaceae presented the greatest number of pollen types. The results demonstrated the species $A$. mellifera $\mathrm{L}$.
\end{abstract}


presents generalist habit, and the families Fabaceae-Mimosoidea, Asteraceae, Commelinaceae and Poaceae are the main pollen sources for A. mellifera in region.

Keywords: honeybee; pollen types; sources pollen.

Recebido em: 23 julho 2013

Aceito em: 06 agosto 2015

\section{Introdução}

As abelhas apresentam uma ampla biodiversidade em todo o mundo, exibindo variados hábitos de vida, desde solitários a sociais ${ }^{(1)}$. O papel ecológico destes insetos é fundamental na manutenção da diversidade vegetal ${ }^{(2)}$, pois eles mantém uma relação intrínseca com inúmeras espécies de plantas ${ }^{(1)}$. Durante suas visitas às flores, as abelhas coletam diferentes materiais desde óleos florais ${ }^{(3)}$, néctar $^{(4,5)}$, resina $^{(6)}$ e pólen ${ }^{(7)}$, do qual obtém recursos para sua sobrevivência, e ainda podem promover a polinização cruzada ao transferirem o pólen de uma flor para outra ${ }^{(2)}$.

Em todas as fases da vida, as abelhas utilizam o pólen e o néctar como principais fontes de alimento. Diferentemente do néctar, o grão de pólen é a principal fonte de nitrogênio para as abelhas, sendo coletado em quantidade nas fontes florais e estocado no alvéolo dentro de seus ninhos para a dieta da colônia $^{(8)}$. Rico em proteínas, apresenta importância vital no desenvolvimento das larvas ${ }^{(9,10)}$; além disso, o pólen também constitui a principal fonte de graxas, vitaminas e sais minerais para as abelhas e a sua ausência pode levar a colmeia à extinção ${ }^{(9,10)}$.

Devido à demanda comercial, a produção de pólen apícola aumentou nos últimos anos e a abelha Apis mellifera destaca-se como a melhor fornecedora deste produto ${ }^{(11)}$. A quantidade de pólen colhido por uma colônia de $A$. mellifera depende da quantidade de operárias forrageiras e da cria (ovos - larvas) presente nesta colônia ${ }^{(12)}$. Nessas condições, o pólen apícola consiste em uma mistura de pólen de flores de várias fontes vegetais coletado pelas abelhas em um determinado local ${ }^{(13)}$.

Os dados sobre fontes de pólen coletadas pelas abelhas geram informações sobre interações ecológicas entre as plantas e seus polinizadores ${ }^{(14)}$. A coleta e a identificação taxonômica de espécies que compõem a fauna e a flora de uma determinada região são informações importantes sobre os recursos naturais nela disponíveis, possibilitando a obtenção de informações básicas para estudos mais amplos sobre as características ecológicas de um determinado habitat ou ecossistema ${ }^{(15)}$. A identificação das espécies apícolas realiza-se a partir de métodos diretos ou indiretos. Os métodos indiretos são basicamente a partir de observações no período de florescimento das espécies vegetais, visando à visitação das abelhas às flores ${ }^{(16)}$. Os métodos indiretos são realizados por meio de análises polínicas dos produtos das abelhas como pólen, mel e própolis.

O presente trabalho teve como objetivo conhecer as fontes de pólen utilizadas por Apis mellifera L. na região semiárida da Bahia.

\section{Material e Métodos}

O trabalho foi realizado em apiário comercial instalado no município de Itaberaba, com as coordenadas $12^{\circ} 31^{\prime} 19$ Sul e $40^{\circ} 18^{\prime} 05$ Oeste, localizado na região do semiárido da Bahia, numa área de caatinga. Coletores de pólen foram instalados na entrada de dez colônias modelo Longstroth, das 18:00 horas do dia anterior até às 18:00 horas do dia da coleta. As coletas de pólen foram realizadas nos meses de dezembro/2006, janeiro, março e dezembro/2007, período em que foi observada florada na região, totalizando 27 amostras.

O material coletado foi pesado e submetido à secagem em estufa com circulação de ar forçado a $60{ }^{\circ} \mathrm{C}$, 
por 72 horas, sendo posteriormente armazenado em recipientes plásticos, devidamente identificados e separados por períodos de coleta. Foram retiradas mensalmente $5 \mathrm{~g}$ de cada amostra individualizada, sendo diluídas em $25 \mathrm{~mL}$ de água destilada a $\left(40^{\circ} \mathrm{C}\right)$, homogeneizadas e colocadas para descansar por duas horas. Retirou-se de cada amostra $2 \mathrm{~mL}$ da solução para desidratação, submetendo-as à centrifugação por 5 minutos a $3000 \mathrm{rpm}$. O sobrenadante foi descartado e o sedimento depositado no fundo do tubo de ensaio foi coletado por meio de um cubo de gelatina glicerinada preso na extremidade de um estilete para a montagem das lâminas ${ }^{(17)}$.

Após a montagem das lâminas, os grãos de pólen foram fotografados em microscópio óptico e analisados qualitativa e quantitativamente.

A análise qualitativa dos tipos polínicos presentes nas amostras foi baseada em literatura especializada, coleções de referência da Palinoteca do Núcleo de Estudos dos Insetos da UFRB e das informações obtidas no campo. A análise quantitativa foi realizada por meio da contagem de 1000 grãos de pólen por lâmina e foram determinadas as seguintes classes de frequência: pólen dominante ( $\mathrm{PD}>45 \%$ ); pólen acessório (PA 16\% a 45\%); pólen isolado importante (PII 3\% a $15 \%$ ) e pólen isolado ocasional (PIO menor que $3 \%)^{(18)}$.

\section{Resultados e Discussão}

Verificou-se nas massas de pólen a presença de 78 tipos polínicos, representados por 24 famílias de plantas, distribuídas nas diferentes classes de ocorrência. Na Tabela 1 estão representados os pólen dominante (PD) e pólen acessório (PA), que apresentaram, respectivamente, um total de 22 e 18 tipos polínicos.

As famílias de plantas que apresentaram maior diversidade de tipos polínicos foram: FabaceaeMimosoidea (12), Asteraceae (11), Commelinaceae (6) e Poaceae (6). Modro et al. ${ }^{(19)}$ encontraram também as famílias Fabaceae e Asteraceae como importantes fornecedoras de fontes polínicas, assim como outros autores têm considerado a família Asteraceae como importante fonte de recursos polínicos para abelhas ${ }^{(20-22)}$.

O elevado potencial apícola da família Fabaceae-Mimosoidea destacou-se devido à sua ampla distribuição no ecossistema e coleta de recursos pelas abelhas eussociais ${ }^{(23,24)}$. De acordo com Freitas $^{(25)}$, espécies de Mimosoidea foram observadas como importantes fontes de pólen para $A$. mellifera, apresentando uma frequência como pólen dominante.

Os tipos polínicos mais constantes nas amostras foram: Asteraceae 2 (83,3\%), Euphorbiaceae (50\%), Hyptis (50\%), Achmella (41,7\%), Jackemontia (41,7\%), Mimosa ophtalmocentra (41,7\%) e Mimosa misera $(41,7 \%)$ (Tabela 1). A presença de plantas da família Asteraceae é comum a outras áreas brasileiras $^{(26)}$.

De acordo com a classe de abundância, foram considerados como PD os tipos polínicos Alternhanthera, Syagrus coronata, Syagrus vagans, Commelina, Croton 2, Mimosa quadrivalvis, Mimosa tenuiflora e Myrcia (Figura 1. A-H). Como PA foram identificados os tipos Alternhanterra tenella, Syagrus 1, Achmella, Bacharris, Conocliniopsis, Heliotropium, Commelina bengalhensis, Jacquemontia, Centrosema sp., Acacia, Mimosa opthalmocentra, M. pudica e Mollugo verticilata.

A análise polínica demonstrou uma elevada participação do PIO em todas as amostras. Segundo alguns autores, produtos das abelhas, quando analisados palinologicamente, caracterizam-se pela grande quantidade de pólen na classe isolado ocasional ${ }^{(27)}$. Estas espécies têm pouca importância quanto à quantidade fornecida, embora seja de interesse na determinação da origem geográfica das amostras. 
Tabela1: Frequência relativa total (FRT \%), Classes de abundância total (CAT) e Constância (C \%) dos tipos polínicos do pólen de Apis mellifera coletados em Itaberaba-BA

\begin{tabular}{|c|c|c|c|c|c|c|c|c|}
\hline \multirow{2}{*}{ Famílias } & \multirow{2}{*}{ Tipos polínicos } & \multicolumn{4}{|c|}{ Período de coleta } & \multirow{2}{*}{ FRT (\%) } & \multirow{2}{*}{ CAT } & \multirow{2}{*}{$\mathbf{C}(\%)$} \\
\hline & & dez/06 & $\mathbf{j a n} / 07$ & $\mathrm{mar} / 07$ & dez/07 & & & \\
\hline \multirow[t]{2}{*}{ Amaranthaceae } & Alternanthera & PD & & $\mathrm{PD}$ & & 5,76 & PD & 16,7 \\
\hline & Alternanthera tenella & & & PD & & 2,29 & PA & 25 \\
\hline Anacardiaceae & Anacardiaceae & & & PD & & 1,35 & PII & 25 \\
\hline \multirow[t]{3}{*}{ Arecaceae } & Syagrus coronata & PD & PD & & PD & 12,82 & PD & 8,3 \\
\hline & Syagrus vagans & & PD & & PD & 8,83 & PD & 8,3 \\
\hline & Syagrus 1 & & & & PD & 1,83 & PA & 8,3 \\
\hline \multirow[t]{5}{*}{ Asteraceae } & Achemella & & PD & & & 1,8 & PA & 41,7 \\
\hline & Asteraceae 1 & & PA & & PA & 0,3 & PII & 83,3 \\
\hline & Baccharis & & PD & & & 2,14 & PA & 25 \\
\hline & Conocliniopsis & & & & PD & 1,57 & PA & 16,7 \\
\hline & Elaphantophus & PA & & & & 0,76 & PIO & 75 \\
\hline Boraginaceae & Heliotropium & & & & PD & 0,17 & PA & 8,3 \\
\hline \multirow[t]{4}{*}{ Commelinaceae } & Commelina 1 & & & & PD & 4,67 & PD & 8,3 \\
\hline & $\begin{array}{l}\text { Commelina } \\
\text { benghalensis }\end{array}$ & PD & & & & 1,65 & PA & 16,7 \\
\hline & Tradeschantia & PA & & & & 0,6 & PIO & 25 \\
\hline & $\begin{array}{l}\text { Tradeschantia } \\
\text { elongata }\end{array}$ & & & & PD & 0,7 & PIO & 8,3 \\
\hline Convolvulaceae & Jacquemontia & PD & & & PA & 1,17 & PA & 41,7 \\
\hline \multirow[t]{3}{*}{ Euphorbiaceae } & Croton 1 & & PA & & & 0,05 & PIO & 16,7 \\
\hline & Croton 2 & PD & & & & 0,78 & PD & 8,3 \\
\hline & Phylantus & & & PA & & 0,28 & PIO & 8,3 \\
\hline \multirow[t]{2}{*}{ Fabaceae } & Fabaceae 2 & & & & PA & 0,6 & PIO & 8,3 \\
\hline & Centrosema sp. & PD & & & & 2,92 & PA & 25 \\
\hline $\begin{array}{c}\text { Fabaceae- } \\
\text { Caesalpinoidea }\end{array}$ & Senna macranthera & & PA & PA & & 0,65 & PII & 16,7 \\
\hline \multirow[t]{9}{*}{ Fabaceae-Mimosoidea } & Acacia & & PD & & & 1,47 & PA & 25 \\
\hline & Mimosaceae 1 & & & PD & & 1,2 & PIO & 33,3 \\
\hline & Mimosa filipis & & & & PA & 1,31 & PII & 8,3 \\
\hline & Mimosa gemulata & & & & PA & 1,25 & PII & 16,7 \\
\hline & $\begin{array}{l}\text { Mimosa } \\
\text { ophthalmocentra }\end{array}$ & & PA & PD & & 1,98 & PA & 41,7 \\
\hline & Mimosa pudica & PD & & & & 1,82 & PA & 16,7 \\
\hline & Mimosa quadrivalvis & & & & PD & 5,78 & $\mathrm{PD}$ & 16,7 \\
\hline & Mimosa tenuiflora & & & PD & PD & 3,37 & PD & 8,3 \\
\hline & Mimosa verrucosa & PD & & & & 1,05 & PIO & 25 \\
\hline Melastomataceae & Melastomataceae & PA & & & & 0,83 & PIO & 8,3 \\
\hline Molluginaceae & Mollugo verticilata & & PD & & & 1,8 & PA & 16,7 \\
\hline Myrtaceae & Myrcia & & PD & PD & & 8,53 & PD & 8,3 \\
\hline \multirow[t]{3}{*}{ Poaceae } & Poaceael & PA & & & & 0,17 & PIO & 8,3 \\
\hline & Poaceae 2 & PD & & & & 0,65 & PII & 16,7 \\
\hline & Poaceae 3 & PA & & & & 0,62 & PIO & 8,3 \\
\hline Solanaceae & Solanum & & & PA & & 0,45 & PIO & 16,7 \\
\hline Sterculiaceae & Sterculiaceae & PA & & & & 0,61 & PIO & 8,3 \\
\hline
\end{tabular}

PD - Pólen Dominante; PA - Pólen Acessório. 


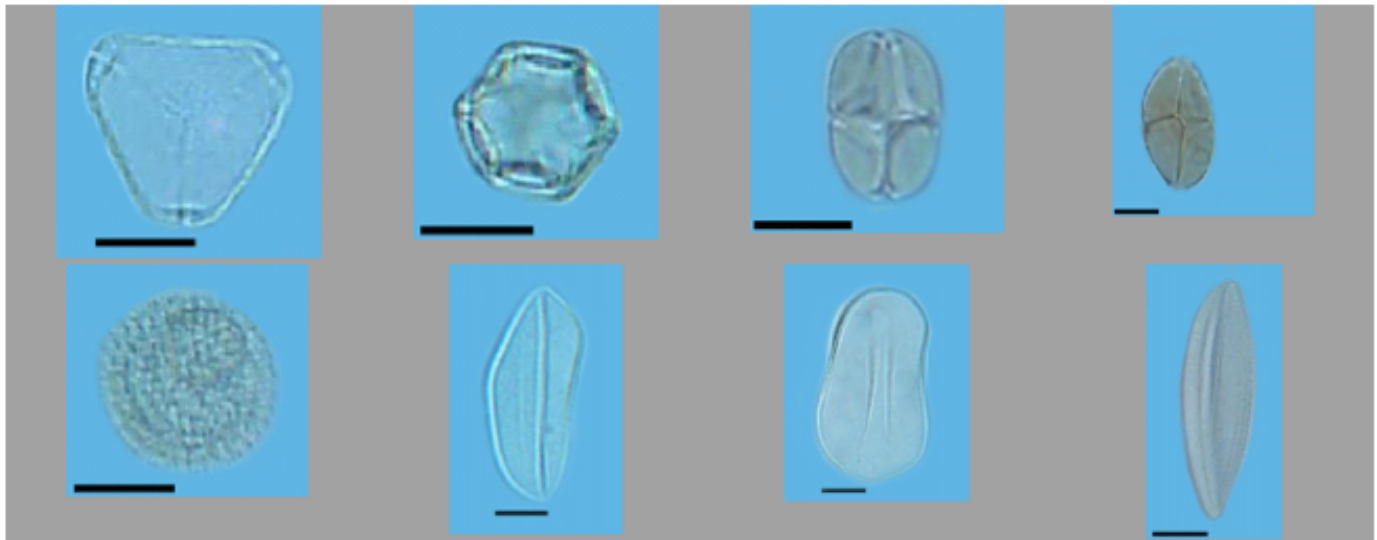

Figura 1: A-H: Tipos polínicos dominantes, observados nas massas de pólen de Apis mellifera L., no município de Itaberaba, Bahia, 2007. A. tipo Myrcia; B. tipo Alternanthera; C. tipo Mimosa quadrivalvis; D. tipo Mimosa tenuiflora; E. tipo Croton 3; F. tipo Syagrus coronata; G. tipo Syagrus vagans; H. tipo Commelina.

\section{Conclusão}

A Apis mellifera L. apresenta comportamento generalista no uso das fontes de pólen, destacando-se as espécies da Familia Fabaceae-Mimosoideae e das famílias Asteraceae, Commelinaceae e Poaceae no município de Itaberaba, região semiárida do Estado da Bahia.

\section{Referências}

1. Imperatriz-Fonseca VL, Nunes-Silva P. As abelhas, os serviços ecossistêmicos e o Código Florestal Brasileiro. Biota Neotropical. 2010;10(4):59-62.

2. Sá NP, Prato M. Conhecendo as abelhas: Um projeto de ensino. Bioscience Journal. 2007;23(1):107-1110.

3. Mendes FN, Rêgo MMC, Albuquerque PMC. Fenologia e biologia reprodutiva de duas espécies de Byrsonima rich. (Malpighiaceae) em área de Cerrado no Nordeste do Brasil. Biota Neotropical. 2011;11(4):103-15.

4. Evangelista-Rodrigues A, Silva EMS, Beserra EMF, Rodrigues ML. Análise físico-química dos méis das abelhas Apis mellifera e Melipona scutellaris produzidos em regiões distintas no Estado da Paraíba. Ciência Rural. 2005;35(5):1166-71.

5. Carvalho-Zilse G, Porto EL, Silva CGN, C. PMdF. Atividade de vôo de operárias de Melipona seminigra (Hymenoptera: Apidae) em um sistema agroflorestal da Amazônia. Bioscience Journal. 2007;23(1):94-9.

6. Manrique AJ, Soares AEE. Início de um programa de seleção de abelhas africanizadas para a melhoria na produção de própolis e seu efeito na produção de mel. Interciencia. 2002;27(6):312-6.

7. Ribeiro EKMD, Rêgo MMC, Machado ICS. Cargas polínicas de abelhas polinizadoras de Byrsonima 
chrysophylla Kunth.(Malpighiaceae): fidelidade e fontes alternativas de recursos florais. Acta Botanica Brasilica. 2008;22(1):165-71.

8. Morgado LN, Andrade RC, Lorenzon MCF, Esteves VG. Padrão polínico utilizado por Tetragonisca angustula Latreille (Apidae: Meliponina). Acta Botanica Brasilica. 2011;25(4):932-4.

9. Ramalho M, Guibu LS, Giannini TC, Kleinert-Giovannini A, Imperatriz-Fonseca VL. Characterization of some southern Brazilian honey and bee plants through pollen analysis. Journal of Apicultural Research. 1991;30:81-6.

10. Bastos EM. Espectro polínico do mel produzido em algumas áreas antrópicas de Minas Gerais. Revista Brasileira de Biologia. 1995;55(4):789-99.

11. Barth OM, Freitas AS, Oliveira ES, Silva RA, Maester FM, Andrella RRS, et al. Evaluation of the botanical origin of commercial dry bee pollen load batches using pollen analysis: a proposal for technical standardization. Anais da Academia Brasileira de Ciências. 2010;82(4):893-902.

12. Pegoraro M, E. N., Chaves Neto A, Fedalto LM. Estoque de recursos alimentares em Apis mellifera scutellata (Hym., Apidae). Archives of Veterinary Science. 1999;4(1):51-6.

13. Oliveira ACd, Valentim IB, Goulart MOF. Fontes vegetais naturais de antioxidantes. Química Nova. 2009;32(3):689-702.

14. Roubik DW. Foraging behavior of competing Africanized honeybees and stingless bees. Ecology. 1980;61:836-45.

15. Prado AP. Importância prática da taxonomia: ou o papel da taxonomia para a entomologia aplicada. Revista Brasileira de Entomologia. 1980;24:165-7.

16. Freitas BM, Silva EMS. Potencial apícola da vegetação do semiárido Brasileiro. In: Santos FAR, editor. Apium Plantae. 3. 1 ed. Recife: Ministério da Ciência e Tecnologia; 2006: 19-32.

17. Alves RMO, Carvalho CAL, Souza BA. Espectro polínico de amostras de mel de Melipona mandacaia Smith, 1863 (Hymenoptera: Apidae). Acta Science Biology Sciences. 2006;28(1):65-70.

18. Louveaux J, Maurizio A, Vorwohl G. Methods of melissopalynology. Bee World. 1978;59(4):139-57.

19. Modro AFH, Marchini LC, Moreti ACdCC. Origem botânica de cargas de pólen de colmeias de abelhas africanizadas em Piracicaba, SP. Ciência Rural. 2011;41(11):1944-51.

20. Ramalho M, Kleinert-Giovannini A, Imperatriz-Fonseca VL. Important bee plants for stingless bees (Melipona and Trigonini) and Africanized honeybees (Apis mellifera) in neotropical habitats: a review. Apidologie. 1990;21:469-88.

21. Marchini LC, Moreti ACCC, Teixeira EW, Silva ECA, Rodrigues RR, Souza VC. Plantas visitadas por abelhas africanizadas em duas localidades do estado de Sao Paulo. Scientia Agrícola. 2001;58:413-20.

22. Marques LJP, Muniz FH, Silva JM. Levantamento apibotânico do município de Santa Luzia do Paruá, Maranhão Resultados preliminares. Revista Brasileira de Biociências. 2007;5(1):114-6.

23. Carvalho CALd, Marques OM, Vidal CA, Neves AMS. Comportamento forrageiro de abelhas (Hymenoptera, Apoidea) em flores de Solanum palinacanthum Dunal (Solanaceae). Revista Brasileira de Zoociências. 2001;3(1):35-444.

24. Lorenzon MCA, Matrangolo CAR, Schoereder JH. Flora visitada pelas abelhas eussociais (Hymenoptera, Apidae) na Serra da Capivara, em Caatinga do Sul do Piauí. Neotropical Entomology. 2003;32(1):27-36.

25. Freitas AS, Arruda VAS, Almeida-Muradian LB, Barth OM. The Botanical Profiles of Dried Bee Pollen Loads Collected by Apis mellifera (Linnaeus) in Brazil. Sociobiology. 2013;60(1):56-64. 
26. Barbola IF, Laroca S, Almeida MC. Utilização de recursos florais por abelhas silvestres (Hymenoptera, Apoidea) da Floresta Estadual Passa Dois (Lapa, Paraná, Brasil). Revista Brasileira de Entomologia. 2000;44:919.

27. Martins ACL, Rêgo MMC, Carreira LMM, Albuquerque PMC. Espectro polínico de mel de tiúba (Melipona fasciculata Smith, 1854, Hymenoptera, Apidae. Acta Amazônica. 2011;41:183-90. 